\title{
Intraoperative Glycaemia Following Paracetamol with and without Glucose: A Randomized-Controlled Trial
}

\author{
Ricardo Mota Pereira ${ }^{1 *}$, Fátima Gonçalves ${ }^{1}$, João Costa ${ }^{2,3,4}$, Filomena Couto $^{5}$, Carolina Sá ${ }^{1}$, \\ Isabel Neves ${ }^{1}$, Lucindo Ormonde ${ }^{1,4}$ \\ ${ }^{1}$ Department of Anaesthesiology, Santa Maria University Hospital, Lisbon, Portugal; ${ }^{2}$ Laboratory of Clinical Pharmacology and \\ Therapeutics, Faculty of Medicine, University of Lisbon, Lisbon, Portugal; ${ }^{3}$ Evidence-Based Medicine Centre, Faculty of Medicine, \\ University of Lisbon, Lisbon, Portugal; ${ }^{4}$ Instituto de Medicina Molecular (IMM), Faculty of Medicine, University of Lisbon, Lisbon, \\ Portugal; ${ }^{5}$ Department of Gynaecology, Santa Maria University Hospital, Lisbon, Portugal. \\ Email: "nricardopereira@gmail.com
}

Received August $3^{\text {rd }}, 2013$; revised August $31^{\text {st }}, 2013$; accepted September $6^{\text {th }}, 2013$

Copyright (C) 2013 Ricardo Mota Pereira et al. This is an open access article distributed under the Creative Commons Attribution License, which permits unrestricted use, distribution, and reproduction in any medium, provided the original work is properly cited.

\begin{abstract}
Background: Hyperglycaemia is conversely a risk factor for perioperative complications. We are currently using a generic $3.3 \mathrm{~g}$ glucose containing formula of intravenous $1000 \mathrm{mg}$ paracetamol for perioperative analgesia. Our main goal was to compare the trends of glycaemic values after administration of a generic $3.3 \mathrm{~g}$ glucose containing formula with a non-glucose containing branded formula of intravenous $1000 \mathrm{mg}$ paracetamol. Methods: A exploratory proof-of-concept randomized clinical trial was conducted with 150 patients scheduled for elective gynaecologic. Patients were randomly assigned into three groups: control group (saline); active-control group: intraoperative administration of a branded non-glucose containing $1000 \mathrm{mg}$ paracetamol formula; experimental group: intraoperative administration of a generic $3.3 \mathrm{~g}$ glucose containing $1000 \mathrm{mg}$ paracetamol formula. The primary outcome was mean change from baseline in glaucoma. In case significant differences were found, the following secondary outcomes were explored: the proportion of patients with high glycaemia values $(>150 \mathrm{mg} / \mathrm{dL})$ and the proportion of patients with negative glycaemic variation. Results: Mean glycaemia change was higher after generic $3.3 \mathrm{~g}$ glucose containing paracetamol formula both in comparison to placebo (16.3 mg/dL [95\% CI: 6.1 to 26.6]) and active-control (19.1 mg/dL [8.2 to 30.0] groups. Similar results were found in the intention-to-treat analysis. In only the experimental group, patients had high glycaemic values (11.3\%). Conclusions: This study showed that in non-diabetic, under non-cardiac surgery, administration of a generic glucose-containing formula of intravenous $1000 \mathrm{mg}$ paracetamol was associated with poorer glycaemic control. These results raise the question of a possible increased risk among these patients. Further studies using diabetic patients are recommended.
\end{abstract}

Keywords: Paracetamol; Hyperglycaemia; Gyneacologic Surgical Procedures; Randomized Controlled Trial

\section{Introduction}

It has been demonstrated that inadequate glycaemic control in surgical patients increases perioperative morbidity and mortality [1]. While most studies focus on neurosurgical [2], cardiac [3-5] and critical care patients [6], and therefore further investigation is required, some of the outcome key findings are most probably applicable to general surgical patients [5].

Acute hyperglycaemia is associated with several deleterious effects such as suppressed immune function, increased systemic vascular resistance, dehydration, electrolyte and acid-base imbalance and central nervous sys-

*Corresponding author. tem dysfunction [7]. Although patients with diabetes mellitus (DM) are at higher risk for perioperative complications [8], the occurrence of intraoperative acute hyperglycaemia in non-DM patients is also considered [9, $10]$ to be a strong and independent predictor of poorer outcome (sepsis, pneumonia, surgical wound infection). Furthermore, individuals with previous unknown hyperglycaemia are at even higher risk than those with prediagnosed DM [2].

Recent studies found that $21 \%$ to $34 \%$ of patients who underwent surgery had uncontrolled blood glucose level (Blood Glycaemia (BG) $>150 \mathrm{mg} / \mathrm{dl}$ ), particularly in the immediate postoperative period ( $<72$ hours) [11]. Many of these patients may miss a DM diagnosis as only two 
thirds of those have a pre-established DM diagnosis [12].

Paracetamol (acetaminophen) is one of the safest and more cost-effective [13] non-opioid analgesic when administered in analgesic doses. Paracetamol is considered an atypical nonsteroidal anti-inflammatory drug (NSAID) [14], given the nonspecific and weak inhibition of COX3 [15], with central and peripheral effects.

In our hospital, it is currently in use a $3 \mathrm{~g}$ glucose containing formula of intravenous $1000 \mathrm{mg}$ paracetamol for perioperative analgesia. To evaluate what is the impact of this glucose containing paracetamol formula in the intraoperative glycaemic control among non-diabetic general surgical patients, we conducted an exploratory proofof-concept randomized clinical trial.

\section{Methods}

All patients provided their written informed consent for participating in this study, which was approved by the Ethics Committee of our Hospital (Santa Maria University Hospital, Lisbon). This was an academic trial without any direct or indirect funding.

\subsection{Study Population}

This study was conducted in the gynaecologic surgery department of Santa Maria University Hospital (Lisbon, Portugal). Non-diabetic female patients scheduled for elective gynaecologic procedure were consecutively recruited. Inclusion criteria were: 1) written informed consent; 2) age between 18 and 80 years-old; 3) body mass index $(\mathrm{BMI})<30 \mathrm{Kg} / \mathrm{m}^{2}$; 4) fasting glycaemic values $>$ $60 \mathrm{mg} / \mathrm{dL}$ and $<126 \mathrm{mg} / \mathrm{dL}$; 5) American Society of Anaesthesiology (ASA) classification $\leq 2$; and 6) 8-hour fasting period. Patients with a diagnosis of DM or glucose impairment were excluded.

\subsection{Study Design}

Patients were randomly assigned to one of three groups: 1) Placebo-control group (saline); 2) Paracetamol 1000 $\mathrm{mg}$ in a non-glucose containing formula (active-control group), and; 3) Paracetamol $1000 \mathrm{mg}$ in a $3 \mathrm{~g}$ glucose containing formula (experimental group). All intervenations were delivered in the last third of the surgical procedure (15 minutes infusion).

Random sequence generation was independently done by the principal investigator (RMP) that did not participate in the patients' recruitment or evaluation. Opaque sealed envelopes in a closed box were used to retain the random codes and successively replaced until 150 consecutive patients were obtained. Hence, each new patient had an equal chance of being allocated to one of the three treatment groups. Allocation concealment was achieved by making patients and assessment investigator (FC) blind to treatment assignment. The investigators (FG, FC) who administered the treatment were the only subjects that were not blind to treatment assignment, however they didn't participated further in the study.

Primary outcome was defined as the mean change from baseline in blood glucose. If significant differences were found between groups, the following secondary outcomes were investigated: proportion of patients with high glycaemia values $(>150 \mathrm{mg} / \mathrm{dL})$ in the second measurement and proportion of patients with negative variation in glycaemia between the two measurements. The same investigator (FC) assessed all patients and performed all measurements. Fingerprick capillary glucose was determined at the beginning of the surgery (baseline glycaemia) and 10 minutes after treatment infusion ending (post-interventional glycaemia), using the Precision Xceed Pro point-of-care glucometer (Abbott), which was calibrated daily. All test-trips used were taken from the same lot. For the capillary glucose measurement, the second finger from the opposite arm in which the drug was administrated, was chosen. In none of the glycemic measurements was found an invalid value, making unnecessary a second attempt.

\subsection{Anaesthesia Protocol}

After an 8-hour preoperative fasting, all patients were pre-medicated with midazolam $0.05 \mathrm{mg} / \mathrm{kg}$. General anaesthesia was induced with single doses of fentanyl (5 $\mathrm{ug} / \mathrm{kg}$ ) and propofol $(2 \mathrm{mg} / \mathrm{kg})$ given slowly as bolus injections. Traqueal intubation was facilitated with rocuronium $(0.4 \mathrm{mg} / \mathrm{kg})$ and anaesthesia maintained with sevoflurane vaporized in air and oxygen mixture $\left(\mathrm{FiO}_{2}\right.$ 0.4) titrated to achieve stable hemodynamics.

\subsection{Statistical Analysis and Data Synthesis}

This was an exploratory trial and we planned to enrol 40 patients per treatment group. Assuming that the response within each subject group is normally distributed with standard deviation (SD) of $20 \mathrm{mg} / \mathrm{dL}$, this sample size will allow detecting a true difference in the mean response of treatment groups (experimental, placebo-control and active-control) of $15 \mathrm{mg} / \mathrm{dL}$ with a probability (power) of $82 \%$ (analysis of variance with Bonferroni correction). We considered that such difference is of potential clinical significance. The Type I error probability associated with this test of the null hypothesis that the population means of the experimental and controls groups were equal was 0.05 .

The mean change from baseline in glycaemia values between groups (primary outcome: dependent variable) was compared using a full factorial generalized linear model (GLM) with treatment group as main effect and including terms for age, fasting period (Nulla per os: NTO), surgery duration and baseline glycaemia. Interac- 
tion terms were then removed depending on their level of non-significance. Type III estimator was used to perform the analysis. In case significant differences were found, Bonferroni post-hoc analysis corrected for multiple comparisons was conducted to explore the nature of such differences.

A per-protocol and an intention-to-treat (ITT) analysis were performed. ITT population comprised all randomized subjects that received treatment. The baseline glycaemia value was used to impute the missing values from patients lacking the second glycaemia measurement.

All statistical analyses were done using SPSS 20.0 for Windows (Lisbon, Portugal). Random codes were broken only after the final results of the statistical analysis.

\section{Results}

Following our inclusion and exclusion criteria, a total of
150 consecutive patients were randomized between January and May 2011. Seventeen patients were excluded from the per-protocol analysis because of missing data from the second glycaemia evaluation $(n=12)$ and deviation of study protocol (the anaesthesiologist did not administered the treatment according to the protocol; $\mathrm{n}=$ 5). As a result, a total of 133 patients were included in the per-protocol analysis (primary analysis) and 145 patients in the ITT analysis. Figure 1 shows the study's flow diagram. The main characteristics of the patients are shown in Table 1.

As a result of the chosen randomization methodology, different groups sizes were obtained (49, 42 and 59 patients, for placebo-control, active-control and experimental groups, respectively) since each new patient had the same probability chance to be included in the different groups.

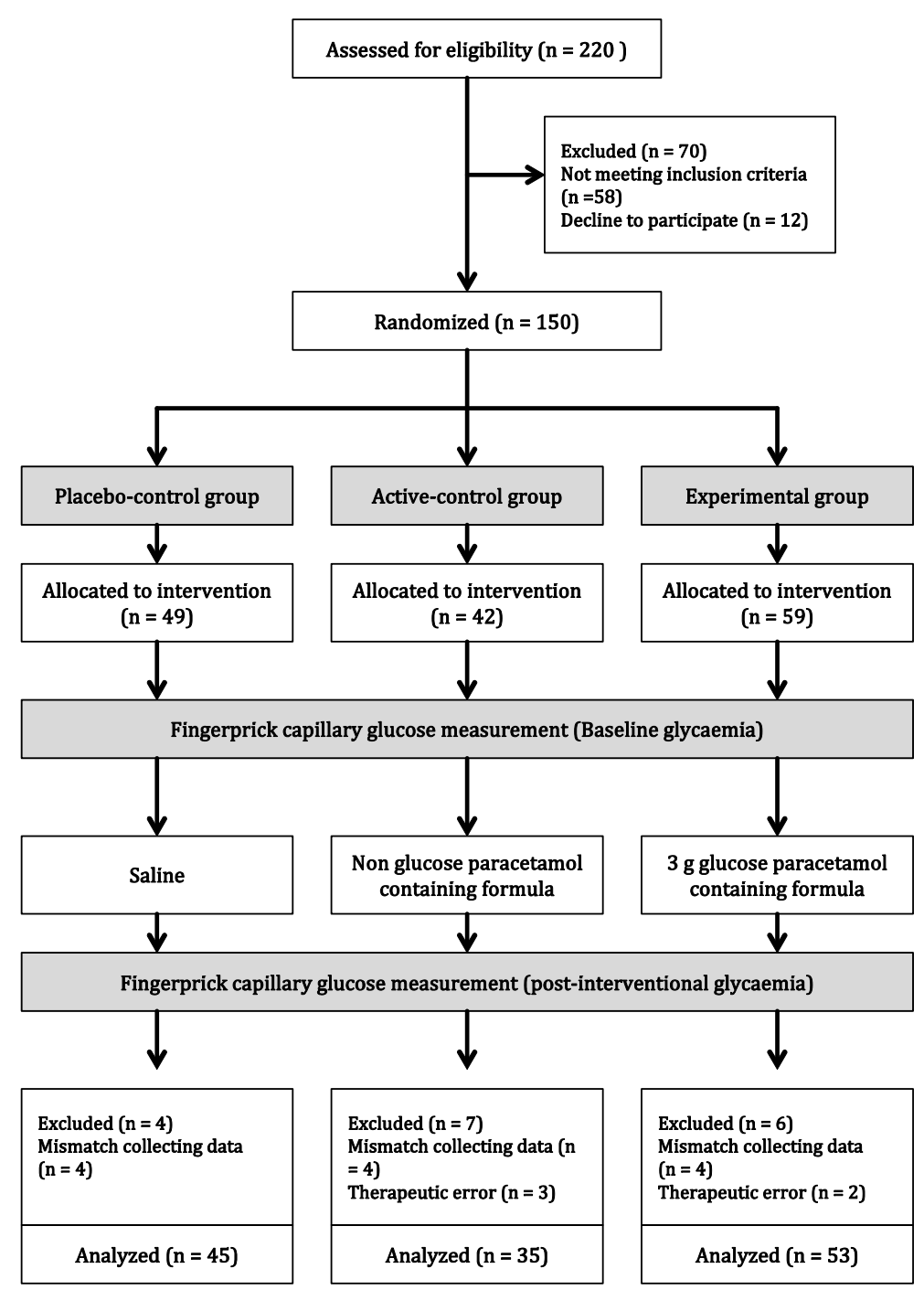

Figure 1. Study flow diagram. Mismatch collecting data due to second glycaemia measurements (lacks of post-interventional glycaemia). Therapeutic error due to intraoperative administration of saline enriched with dextrose. 
Table 1. Characteristics of the patients.

\begin{tabular}{ccccc}
\hline & $\begin{array}{c}\text { Placebo (Saline) } \\
\text {-Control Group }\end{array}$ & $\begin{array}{c}\text { Paracetamol } 1 \mathrm{~g} \text { in non-glucose formula } \\
\text { (Active - Control Group) }\end{array}$ & $\begin{array}{c}\text { Paracetamol } 1 \mathrm{~g} \text { in } 3 \mathrm{~g} \text { glucose } \\
\text { formula }\end{array}$ & $\mathrm{p}$ value \\
\hline $\mathrm{n}$ (ITT population) & 49 & 39 & 57 & $45.7 \pm 1.7$ \\
& $40.3 \pm 1.9$ & $48.3 \pm 2.1$ & $(23$ to 78$)$ & 1.7 \\
Age, (years) & $(18$ to 68$)$ & $99 \pm 7$ to 81$)$ & $(28$ to 295$)$ & 0.014 \\
& $103 \pm 7$ & $(20$ to 230$)$ & $11.1 \pm 0.3$ & $(8$ to 15$)$ \\
Surgery duration (min) & $(35$ to 245$)$ & $10.2 \pm 0.4$ & $85.9 \pm 1.7$ & 0.091 \\
& $11.4 \pm 0.4$ & $(8$ to 17$)$ & $(68$ to 128$)$ & 0.628 \\
NPO (hours) & $(8$ to 18$)$ & $91.1 \pm 2.0$ & & \\
Baseline glycemia $(\mathrm{C} 1)$ & $83.9 \pm 1.8$ & $(74$ to 114$)$ & & \\
$(\mathrm{mg} / \mathrm{dL})$ & $(73$ to 125$)$ & &
\end{tabular}

Legend: Data expressed as Mean \pm Standard Error $(\mathrm{SE}) . \mathrm{NPO}=$ Nulla per os.

No significant interactions existed between treatment, surgery time, NPO and baseline glycaemia. Placebo control group patients were younger than in the other groups $(p=0.014)$. Table 2 shows the main results for the perprotocol and ITT analysis of the primary outcome (mean change from baseline in blood glucose), as well as the results of the exploratory secondary outcomes. Post-hoc analysis for the primary outcome showed significant differences between the experimental group and both placebo and active-control groups ( $\mathrm{p} \leq 0.001$ for both perprotocol and ITT data), without significant differences between the placebo and the active-control group. For the per-protocol population, the mean difference between the experimental and the placebo and active-control groups were $16.3 \mathrm{mg} / \mathrm{dL}$ (95\% Confidence Interval [CI]: 6.1 to $26.6)$ and $19.1 \mathrm{md} / \mathrm{dL}$ (95\% CI: 8.2 to 30.0 ), respectively. For the ITT population, these differences were 15.0 $\mathrm{md} / \mathrm{dL}(95 \%$ CI: 5.2 to 24.7$)$ and $18.4 \mathrm{md} / \mathrm{dL}(95 \% \mathrm{CI}$ : 7.9 to 28.8 ), respectively.

In only the experimental group, patients $(11.3 \%)$ had high glycaemia values (Figure 2). All glycaemic measurements were above threshold for hypoglycaemia (minimum glucose value was $65 \mathrm{mg} / \mathrm{dL}, 74 \mathrm{mg} / \mathrm{dL}$ and 66 $\mathrm{mg} / \mathrm{dL}$ for placebo-control, active-control and experimental groups, respectively).

\section{Discussion}

The most relevant findings of our study are: 1) intravenous paracetamol formulation containing glucose is associated with a mean increase of glycaemia in female non-diabetic patients submitted to elective gynaecological surgery; Similar results were found considered both per-protocol and ITT analysis, which strength this conclusions; 2) intravenous paracetamol formulation containing glucose is associated with a higher proportion of patients showing poor glycaemic control. In fact, only patients randomised to the experimental group had absolute glucose blood values above the so-called "hyperglycaemic barrier" (>150 - $180 \mathrm{mg} / \mathrm{dl})$.
As previously described, high glycaemic values might increase the risk of occurrence of adverse effects and poor outcomes after surgery. The clinical association between hyperglycaemia and adverse clinical outcomes was first reported in 1985 when Longstreth and Inui [4] demonstrated a poorer neurologic recovery following out-of-hospital cardiac arrest associated with hyperglycemia. Several mechanisms promote hyperglycaemia as a response to the metabolic stress during surgery, namely through the production and release of the counterregulatory hormones (glucagon, epinephrine and cortisol) [7], peripheral insulin resistance throughout glucocorticoid therapy [2] and glucose-stimulated insulin depression by inhalatory anesthesic agents. Although patients with DM have a higher incidence of perioperative complications $[16,17]$, development of acute hyperglycaemia perioperatively per se (i.e. even in those with previously normal glucose tolerance) is also recognized as a predictor of adverse outcome. [7,18]

The goal of optimal glycaemic values has been the subject of several studies. [19-21] Nevertheless, the glycaemic values considered as optimal for medical and surgical patients have been controversial, both for the intra- or perioperative periods and for critical or noncritical patients. Van der Berghe et al. reported the first major trial of intensive insulin therapy (IIT) in an adult critical care unit. By targeting blood glucose $<110 \mathrm{mg} / \mathrm{dl}$, they reported a $32 \%(95 \% \mathrm{CI}: 2 \%-55 \%)$ risk reduction in mortality, particularly from multiple organ failure and sepsis. [22] Unfortunately, several subsequent multiinstitutional studies have failed to replicate these results. The NICE-SUGAR (Normoglycaemia in Intensive Care Evaluation-Survival Using Glucose Algorithm Regulation) reported an increase risk of death at 90 days (Odds Ratio 1.14; 95\% CI: 1.02 - 1.28) with IIT strategy to achieve $81-108 \mathrm{mg} / \mathrm{dl}$ compared with a more relaxed target $(<180 \mathrm{mg} / \mathrm{dl})$. According to the authors, this mortality increase was related with the higher risk of hypoglycaemia episodes. [23]

Although there's controversy between the benefits of 
Table 2. Outcome results.

\begin{tabular}{|c|c|c|c|c|c|}
\hline Outcome & Population & $\begin{array}{l}\text { Placebo (Saline) } \\
\text {-Control Group }\end{array}$ & $\begin{array}{c}\text { Paracetamol 1g in } \\
\text { non-glucose formula } \\
\text { (Active - Control Group) }\end{array}$ & $\begin{array}{l}\text { Paracetamol } 1 \mathrm{~g} \text { in } 3 \mathrm{~g} \\
\text { glucose formula }\end{array}$ & $\mathrm{p}$ value \\
\hline \multirow{2}{*}{$\begin{array}{l}\text { Primary outcome: Mean } \\
\text { change from baseline in } \\
\text { blood glucose }(\mathrm{mg} / \mathrm{dL})\end{array}$} & $\begin{array}{l}\text { Per-Protocol analysis } \\
\qquad(\mathrm{n}=133)\end{array}$ & $\begin{array}{l}17.5 \pm 3.1 \\
(-7 \text { to } 65)\end{array}$ & $\begin{array}{l}14.7 \pm 3.5 \\
(-10 \text { to } 50)\end{array}$ & $\begin{array}{l}33.8 \pm 2.8 \\
(-9 \text { to } 116)\end{array}$ & $\begin{array}{l}F=6.357 \\
p<0.001\end{array}$ \\
\hline & $\begin{array}{l}\text { ITT analysis } \\
(\mathrm{n}=145)\end{array}$ & $16.3 \pm 3.0$ & $13.0 \pm 3.4$ & $31.3 \pm 2.7$ & $\begin{array}{l}F=6.434 \\
p<0.001\end{array}$ \\
\hline $\begin{array}{l}\mathrm{N}(\%) \text { with high glycemia } \\
(>150 \mathrm{mg} / \mathrm{dL})\end{array}$ & $\begin{array}{l}\text { Per-Protocol analysis } \\
\qquad(\mathrm{n}=133)\end{array}$ & $0(0)$ & $0(0)$ & $6(11.3)$ & $0.009^{*}$ \\
\hline $\begin{array}{l}\mathrm{N}(\%) \text { with negative } \\
\text { glycaemia variation }\end{array}$ & $\begin{array}{l}\text { Per-Protocol analysis } \\
\qquad(\mathrm{n}=133)\end{array}$ & $5(11.1)$ & $5(14.3)$ & $2(3.8)$ & $0.198^{*}$ \\
\hline
\end{tabular}

Legend: Data expressed as Mean \pm Standard Error (SE). ${ }^{*}$ Fisher exact test.
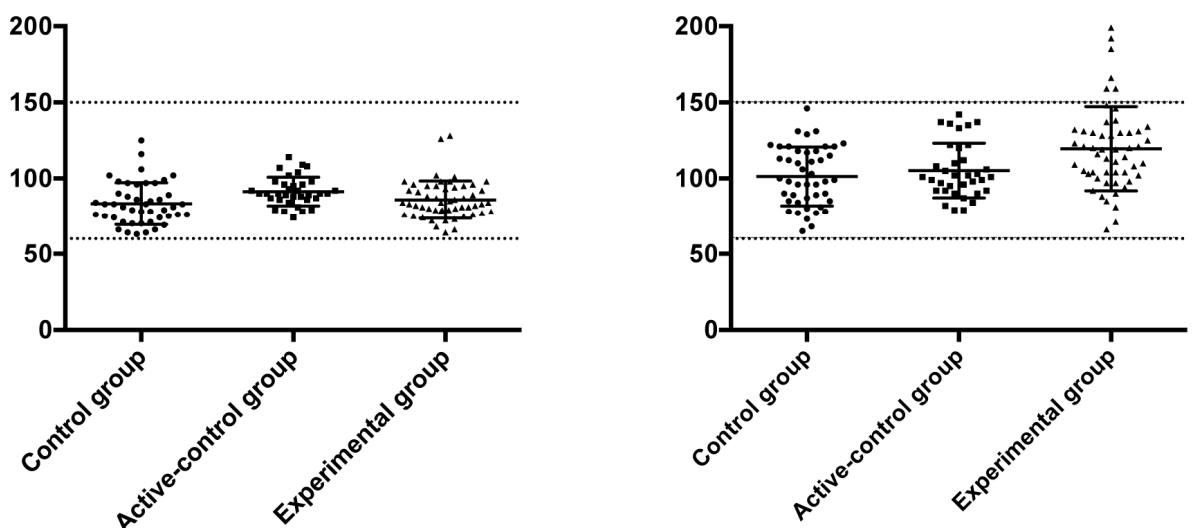

Figure 2. Baseline and post-intervention glycaemia for individual patients, according to treatment group. Values are expressed in $\mathrm{mg} / \mathrm{dL}$.

tight blood glucose control $(<110 \mathrm{mg} / \mathrm{dl})$ versus the standard blood glucose management $(<200 \mathrm{mg})$, there is some data [24] suggesting that glycaemic values above $140 \mathrm{mg} / \mathrm{dl}$ are associated with post-operative complications and poorer outcome. In fact, randomized controlled trials in medical [23], cardiac [25-27] and neurosurgical [28] populations have found reduced rates of bacteraemia, duration of antibiotic usage, infections rates [29], and incidence of recurrent infections in patients with tight glycaemic control ( $<150 \mathrm{mg} / \mathrm{dl})$ [1]. Margarita Ramos et al. further showed that every $40 \mathrm{mg} / \mathrm{dL}$ increase in postoperative glucose above those values led to a $30 \%$ increased risk of postoperative infections (pneumonia, wound infections, urinary tracts infections and sepsis) in the first 30 days after surgery [30].

Most of the research on glucose control has been conducted in the critical care setting and the results obtained, being later generalized to the non-critical and non-cardiac surgery patients [1,31]. In 2010, the Society for Ambulatory Anaesthesia (SAMBA) [32] published guidelines for perioperative management in diabetic patients undergoing ambulatory surgery. According to this guide- line, in patients with well-controlled diabetes the intraoperative blood glucose levels should be maintained below $180 \mathrm{mg} / \mathrm{dl}$. Guidelines for non-critical and non-DM patients are still lacking. Our results are a first contribution for an evidence-based discussion on this matter.

In addition, perioperative glycaemic control also depends on hypoglycaemia prevention, namely due to potential neurological injury. [33,34] A blood glucose level below $70 \mathrm{mg} / \mathrm{dl}$ is generally considered an alert value for hypoglycaemia. [35] This end point value allows time for prevention of symptomatic hypoglycaemia, which usually occurs at blood glucose levels of 45 to $55 \mathrm{mg} / \mathrm{dL}$. [32] During our study, no patients showed hypoglycaemia.

It is predictable that any anaesthetic technique that modifies the intra-operatively neuroendocrine stress response could also modulate the subsequent metabolic sequelae and mitigate perioperative hyperglycaemia. [7] It is well known the benefit effect of blocking the sympathetic neuroendocrine response through the spinal and epidural anaesthesic techniques on the preventive strategy of supressing the hyperglycaemic stimulus [36]. Dif- 
ferently, propofol and opioids do not have a major effect on glucose metabolism. [2] In this study, all patients were submitted to surgery under balanced general anaesthesia and with the same anaesthetic protocol, according to patients' weight. Therefore, our study is not likely biased due to confounding anaesthesic variables.

A variety of measurement techniques are currently in use. Arterial samples are considered more accurate [37]. It is known that capillary testing should be avoided in patients with haematocrit levels $<25 \%$ or $>60 \%$, in shock, with severe dehydration and when vasoactive agents such as norepinephrine are given [38-40]. However, for most surgical patients, phlebotomy or arterial access is not routine [41]. In hemodynamically stable patients the capillary glucose meter correlates well with laboratory reference values [7] and is strongly correlated with arterial samples [41]. Also, the laboratory plasma values generally provide little additional information for non-ICU patients and typically lower the mean glucose [42]. In this study, all patients were hemodynamically stable and non-critically ill, submitted to minor/moderate gynaecologic procedures. Therefore, we think that the glycaemia measurement technique used in our study does not represent a major limitation. Furthermore, to minimize bias, the same investigator using the same capillary glucose meter and technique, performed all measurements.

In our hospital, paracetamol is included in the analgesic strategy for all patients (medical and surgical). Several peripheral and central mechanisms of action have been suggested to explain the paracetamol analgesic properties including selective inhibition of cyclooxygenase activity in the CNS, spinal interaction with 5-HT3 receptors [14], inhibition of neurons excited by substance $\mathrm{P}$ and activation of suprasegmental descending inhibitory pathways [15]. Due to the analgesic properties, paracetamol plays a key role in suppressing the surgery-induced pain adrenergic stimulation, and this suppression could, in theory, blunt the adrenergic hyperglycaemic response. In our opinion, this is the most plausible explanation for the findings in the paracetamol (without glucose) group, which had the lowest increase in glycaemia.

Finally, as with most studies, this also presented some limitations. First, this was an exploratory trial, which aimed to address the question if paracetamol with glucose is associated with an increased risk of poorer glycaemic control. We have not evaluated the clinical consequences of this poorer glycaemic control. Second, sample size was relatively small and we included only female patients undergoing minor to moderate gynaecologic procedures. This precludes the external validity of the findings to other non-diabetic populations and gender. Third, female patients underwent different types of surgeries. We have not performed post-hoc subgroup analysis because of low power. The possibility exists that the results could also differ according to the type of surgery. Fourth, the paracetamol brand used in the experimental $\left(\right.$ Paracetamol-APS $\left.^{\circledR}\right)$ and active-control $\left(\right.$ Perfalgan $\left.^{\circledR}\right)$ groups were different. Although these are thought to be equivalent, we cannot rule out bias emerging from this difference.

\section{Conclusion}

In conclusion, our results strongly suggest that administration of a glucose containing formula of paracetamol may increase the risk of perioperative hyperglycemia in non-diabetic patients submitted to non-cardiac surgery. However, this was an exploratory "proof-of-concept" trial and, although the results were robust, conclusions and implications for practice should be thought with caution. Future clinical research should address the postoperative outcomes and potential consequences of this poorer glycaemic control.

\section{Acknowledgements}

Specific author contributions: RMP contributed to the concept and design, data analysis, and interpretation of the data; wrote the first draft of the manuscript; critically revised the manuscript; and gave final approval of the submitted manuscript. FG contributed to the concept, design, and data acquisition; critically revised the manuscript; and gave final approval of the submitted manuscript. JC contributed to data analysis, and interpretation of the data; critically revised the manuscript; and gave final approval of the submitted manuscript. IN made contribution in the concept and design; and gave final approval of the submitted manuscript. FC contributed to data acquisition. LO gave final approval of the submitted manuscript.

Financial support and sponsorship: This was an academic project without any direct or indirect funding.

Conflicts of interest: The authors do not have any potential conflict of interest to declare.

Acknowledgement: Cochrane Coordinating Centre in Portugal.

\section{REFERENCES}

[1] T. A. Raju, M. C. Torjman and M. E. Goldberg, "Perioperative Blood Glucose Monitoring in the General Surgical Population," Journal of Diabetes Science and Technology, Vol. 3 No. 6, 2009, pp. 1282-1287.

[2] D. A. Godoy, M. Di Napoli, A. Biestro and R. Lenhardt, "Perioperative Glucose Control in Neurosurgical Patients," Anesthesiology Research and Practice, 2013, 1-13.

[3] P. Lecomte, L. Foubert, F. Nobels, et al., "Dynamic Tight Glycemic Control During and after Cardiac Surgery Is Effective, Feasible, and Safe," Anesthesia \& Analgesia, Vol. 107, No. 1, 2008, pp. 51-58. 


\section{doi:10.1213/ane.0b013e318172c557}

[4] W. T. Longstreth and T. S. Inui, "High Blood Glucose Level on Hospital Admission and Poor Neurological Recovery after Cardiac Arrest," Annals of Neurology, Vol. 15, No. 1, 1984, pp. 59-63. doi:10.1002/ana.410150111

[5] A. E. Duncan, A. Abd-Elsayed, A. Maheshwari, M. Xu, E. Soltesz and C. G. Koch, "Role of Intraoperative and Postoperative Blood Glucose Concentrations in Predicting Outcomes after Cardiac Surgery," Anesthesiology, Vol. 112, No. 4, 2010, pp. 860-871. doi:10.1097/ALN.0b013e3181d3d4b4

[6] D. Lena, P. Kalfon, J.-C. Preiser and C. Ichai, "Glycemic Control in the Intensive Care Unit and during the Postoperative Period," Anesthesiology, Vol. 114, No. 2, 2011, pp. 438-444. doi:10.1097/ALN.0b013e3182078843

[7] S. Akhtar, P. G. Barash and S. E. Inzucchi, "Scientific Principles and Clinical Implications of Perioperative Glucose Regulation and Control," Anesthesia \& Analgesia, Vol. 110, No. 2, 2010, pp. 478-497. doi:10.1213/ANE.0b013e3181c6be63

[8] O. Alexandre, P. Lecomte and Y. Le Manach, "Poor Intraoperative Blood Glucose Control Is Associated with a Worsened Hospital Outcome after Cardiac Surgery in Diabetic Patients," Anesthesiology, Vol. 103, No. 4, 2010, pp. 687-694.

[9] G. V. Bochicchio, L. Salzano, M. Joshi, K. Bochicchio and T. M. Scalea, "Admission Preoperative Glucose Is Predictive of Morbidity and Mortality in Trauma Patients Who Require Immediate Operative Intervention," The American Journal of Surgery, Vol. 71, No. 2, 2005, pp. 171-174.

[10] G. V. Bochicchio, J. Sung, M. Joshi, et al., "Persistent Hyperglycemia Is Predictive of Outcome in Critically Ill Trauma Patients," Journal of Trauma, Vol. 58, No. 5, 2005, pp. 921-924. doi:10.1097/01.TA.0000162141.26392.07

[11] S. Ganai, M. K. F. Lee, et al., "Adverse Outcomes of Geriatric Patients Undergoing Abdominal Surgery Who Are at High Risk for Delirium," Archives of Surgery, Vol. 142, No. 11, 2007, pp. 1072-1078. doi:10.1001/archsurg.142.11.1072

[12] G. E. Umpierrez, S. D. Isaacs, N. Bazargan, X. You, L. M. Thaler and A. E. Kitabchi, "Hyperglycemia: An Independent Marker of In-Hospital Mortality in Patients with Undiagnosed Diabetes," The Journal of Clinical Endocrinology \& Metabolism, Vol. 87, No. 3, 2002, pp. 978982. doi: $10.1210 /$ jc. 87.3 .978

[13] E. P. Krenzelok and M. A. Royal, "Confusion: Acetaminophen Dosing Changes Based on NO Evidence in Adults," Drugs in R\&D, Vol. 12, No. 2, 2012, pp. 45-48

[14] K. Toussaint, X. C. Yang, M. A. Zielinski, et al., "What Do We (Not) Know about How Paracetamol (Acetaminophen) Works?" Journal of Clinical Pharmacy and Therapeutics, Vol. 35, No. 6, 2010, pp. 617-638. doi:10.1111/j.1365-2710.2009.01143.x

[15] H. F. Miranda, M. M. Puig, J. C. Prieto and G. Pinardi, "Synergism between Paracetamol and Nonsteroidal AntiInflammatory Drugs in Experimental Acute Pain," Pain, Vol. 121, No. 1, 2006, pp. 22-28. doi:10.1016/j.pain.2005.11.012

[16] S. E. Siegelaar, J. Hermanides, H. M. Oudemans-van Straaten, et al., "Mean Glucose during ICU Admission Is Related to Mortality by a U-Shaped Curve in Surgical and Medical Patients: A Retrospective Cohort Study," Critical Care, Vol. 14, No. 6, 2010, p. R224. doi:10.1186/cc9369

[17] G. E. Umpierrez, D. Smiley, A. Zisman, et al., "Randomized Study of Basal-Bolus Insulin Therapy in the Inpatient Management of Patients with Type 2 Diabetes (RA BBIT 2 Trial)," Diabetes Care, Vol. 30, No. 9, 2007, pp. 2181-2186. doi:10.2337/dc07-0295

[18] R. Hirose, F. Xu, K. Dang, et al., "Transient Hyperglycemia Affects the Extent of Ischemia-Reperfusion-Induced Renal Injury in Rats," Anesthesiology, Vol. 108, No. 3, 2008, pp. 402-414. doi:10.1097/ALN.0b013e318164cff8

[19] A. M. Sheehy and R. A. Gabbay, "An Overview of Preoperative Glucose Evaluation, Management, and Perioperative Impact," Journal of Diabetes Science and Technology, Vol. 3, No. 6, 2009, pp. 1261-1269.

[20] L. F. Meneghini, "Perioperative Management of Diabetes: Translating Evidence into Practice," Cleveland Clinic Journal of Medicine, Vol. 76, No. 4, 2009, pp. S53-S59. doi:10.3949/ccjm.76.s4.09

[21] A. Gautnam, A. Balusch, A. D. Kaye and E. A. Frost, "Modern Strategies for the Anesthesic Management of the Patient with Diabetes," M.E.J. Anesthesia, Vol. 20, No. 2, 2009, pp. 187-197.

[22] G. Van Den Berghe, P. Wouters, F. Weekers and C. Verwaest, "Intensive Insulin Therapy in Critically Ill Patients," The New England Journal of Medicine, Vol. 345, No. 19, 2001, pp. 1356-1367.

[23] S. Finfer, FRCP, FJFICM, et al., "Intensive versus Conventional Glucose Control in Critically Ill Patients," The New England Journal of Medicine, Vol. 360, No. 13, 2009, pp. 1283-1297. doi:10.1056/NEJMoa0810625

[24] E. S. Moghissi, M. T. Korytkowski, M. Di Nardo, et al., "American Association of Clinical Endocrinologists and American Diabetes Association Consensus Statement on Inpatient Glycemic Control," Diabetes Care, Vol. 32, No. 6, 2009, pp. 1119-1131. doi:10.2337/dc09-9029

[25] J. Steven and S. Nicolson, "Perioperative Management of Blood Glucose during Open Heart Surgery in Infants and Children," Pediatric Anesthesia, Vol. 21, No. 5, 2011, pp. 530-537. doi:10.1111/j.1460-9592.2011.03587.x

[26] G. Y. Gandhi, G. A. Nuttall, M. D. Abel, et al., "Intraoperative Hyperglycemia and Perioperative Outcomes in Cardiac Surgery Patients," Mayo Clinic Proceedings, Vol. 80, No. 7, 2005, pp. 862-866. doi:10.4065/80.7.862

[27] F. Puskas, H. P. Grocott, W. D. White, J. P. Mathew, M. F. Newman and S. Bar-Yosef, "Intraoperative Hyperglycemia and Cognitive Decline after CABG," The Annals of Thoracic Surgery, Vol. 84, No. 5, 2007, pp. 1467-1473. doi:10.1016/j.athoracsur.2007.06.023

[28] F. Bilotta and G. Rosa, "Glucose Management in the Neurosurgical Patient: Are We yet Any Closer?" Current Opinion in Anaesthesiology, Vol. 23, No. 5, 2010, pp. 539-543. doi:10.1097/ACO.0b013e32833e150a 
[29] L. S. Kao, D. Meeks, V. A. Moyer and K. P. Lally "PeriOperative Glycaemia Control Regimens for Preventing Surgical Site Infection in Adults," Cochrane Database of Systematic Reviews, John Wiley \& Sons, Ltd, New York, p. 12.

[30] M. Ramos, Z. Khalpey, S. Lipsitz, et al., "Relationship of Perioperative Hyperglycemia and Postoperative Infections in Patients Who Undergo General and Vascular Surgery," Transactions of the Meeting of the American Surgical Association, Vol. 126, 2008, pp. 228-234. doi:10.1097/SLA.0b013e31818990d1

[31] A. K. M. Lipshutz and M. A. Gropper, "Perioperative Glycemic Control: An Evidence-Based Review," Anesthesiology, Vol. 110, No. 2, 2009, pp. 408-421.

[32] G. P. Joshi, F. Chung, M. A. Vann, et al., "Society for Ambulatory Anesthesia Consensus Statement on Perioperative Blood Glucose Management in Diabetic Patients Undergoing Ambulatory Surgery," Anesthesia \& Analgesia, Vol. 111, No. 6, 2010, pp. 1378-1387. doi:10.1213/ANE.0b013e3181f9c288

[33] D. Kansagara, R. Fu, M. Freeman, F. Wolf and M. Helfand, "Intensive Insulin Therapy in Hospitalized Patients: A Systematic Review," Annals of Internal Medicine, Vol. 154, No. 4, 2011, pp. 268-282. doi:10.7326/0003-4819-154-4-201102150-00008

[34] C. Ryan, A. Vega and A. Drash, "Cognitive Deficits in Adolescents Who Developed Diabetes Early in Life," $\mathrm{Pe}$ diatrics, Vol. 75, No. 5, 1985, pp. 921-927.

[35] P. E. Cryer, L. Axelrod, A. B. Grossman, S. R. Heller, V. M. Montori, E. R. Seaquist and F. J. Service "Evaluation and Management of Adult Hypoglycemic Disorders: An Endocrine Society Clinical Practice Guidelines," The Journal of Clinical Endocrinology \& Metabolism, Vol. 94, 2009, pp. 709-728.

[36] C. H. Jensen, P. Berthelsen, C. Kühl and H. Kehlet, "Ef- fect of Epidural Analgesia on Glucose Tolerance during Surgery," Acta Anaesthesiologica Scandinavica, Vol. 24, No. 6, 1908, pp. 472-474. doi:10.1111/j.1399-6576.1980.tb01586.x

[37] N. K. Skjaervold, E. Solligård, D. R. Hjelme and P. Aadahl, "Continuous Measurement of Blood Glucose: Validation of a New Intravascular Sensor," Anesthesiology, Vol. 114, No. 1, 2011, pp. 120-125. doi:10.1097/ALN.0b013e3181ff4187

[38] H. F. Pidcoke, C. E. Wade, E. A. Mann, et al., "Anemia Causes Hypoglycemia in Intensive Care Unit Patients Due to Error in Single-Channel Glucometers: Methods of Reducing Patient Risk," Critical Care Medicine, Vol. 38, No. 2, 2010, pp. 471-476. doi:10.1097/CCM.0b013e3181bc826f

[39] E. A. Mann, J. Salinas, H. F. Pidcoke, S. E. Wolf, J. B. Holcomb and C. E. Wade, "Error Rates Resulting from Anemia Can Be Corrected in Multiple Commonly Used Point-of-Care Glucometers," Journal of Trauma, Vol. 64, No. 1, 2008, pp. 15-20.

[40] M. J. Rice, A. D. Pitkin and D. B. Coursin, "Glucose Measurement in the Operating Room: More Complicated than It Seems," Anesthesia \& Analgesia, Vol. 110, No. 1, 2010, pp. 1058-1065.

[41] F. Akinbami, S. Segal, J. L. Schnipper, M. StopfkuchenEvans, J. Mills and S. O. Rogers, "Tale of Two Sites: Capillary versus Arterial Blood Glucose Testing in the Operating Room," AJS, Vol. 203, No. 4, 2012, pp. 423427.

[42] J. L. Schnipper, M. Magee, K. Larsen, S. E. Inzucchi and G. Maynard, "Society of Hospital Medicine Glycemic Control Task Force Summary: Practical Recommendations for Assessing the Impact of Glycemic Control Efforts," Journal of Hospital Medicine, Vol. 3, No. S5, 2008, \#p. 66-75. doi:10.1002/jhm.356 